\title{
A compact steep spectrum radio source in NGC 1977 (Research Note)
}

\author{
D. A. Roshi ${ }^{1,2}$ and S. M. Ransom ${ }^{1}$ \\ 1 National Radio Astronomy Observatory ${ }^{\star}$, Charlottesville, VA 22903-4608, USA \\ e-mail: [aroshi; sransom] @nrao.edu \\ 2 National Radio Astronomy Observatory, Green Bank, WV 24944, USA \\ Received 23 May 2012 / Accepted 5 September 2012
}

\section{ABSTRACT}

\begin{abstract}
Context. A compact steep spectrum radio source (J0535-0452) is located in the sky coincident with a bright optical rim in the H II region NGC 1977. J0535-0452 is observed to be $\leq 100$ mas in angular size at $8.44 \mathrm{GHz}$. The spectrum for the radio source is steep and straight with a spectral index of -1.3 between 330 and $8440 \mathrm{MHz}$. No $2 \mu \mathrm{m}$ IR counter part for the source is detected. These characteristics indicate that the source may be either a rare high redshift radio galaxy or a millisecond pulsar (MSP).

Aims. We investigate whether the steep spectrum source is a millisecond pulsar. The optical rim is believed to be the interface between the $\mathrm{H}$ in region and the adjacent molecular cloud. If the compact source is a millisecond pulsar, it would have eluded detection in previous pulsar surveys because of the extreme scattering due to the $\mathrm{H}_{\text {II }}$ region-molecular cloud interface.

Methods. The limits obtained on the angular broadening along with the distance to the scattering screen are used to estimate the pulse broadening. The pulse broadening is shown to be less than a few msec at frequencies $\gtrsim 5 \mathrm{GHz}$. We therefore searched for pulsed emission from J0535-0452 at 14.8 and $4.8 \mathrm{GHz}$ with the Green Bank Telescope (GBT).

Results. No pulsed emission is detected to 55 and $30 \mu \mathrm{Jy}$ level at 4.8 and $14.8 \mathrm{GHz}$. Based on the parameter space explored by our pulsar search algorithm, we conclude that, if J0535-0452 is a pulsar, then it could only be a binary MSP of orbital period $\lesssim 5 \mathrm{~h}$.
\end{abstract}

Key words. pulsars: general - HII regions - scattering

\section{Introduction}

NGC 1977 is an H II region located at the northern edge of the Orion molecular cloud at a distance of about $0.5 \mathrm{kpc}$ from the Sun. The region has an emission measure of $5 \times 10^{3} \mathrm{pc} \mathrm{cm}^{-6}$ and is believed to be an example of an interface between the $\mathrm{H}$ II region and the adjacent molecular cloud (Shaver \& Goss 1970). A 6-cm VLA image of the H II region made by Kutner et al. (1985) shows a strong peak that coincides with the brightest part of the optical bright rim. They investigated whether the radio peak is due to a compact $\mathrm{H}$ II region or dense clump of gas ionized by the central star in NGC 1977. Both these possibilities were ruled out based on the expected radio and IR emission from such ionized gas. Subrahmanyan et al. (2001) imaged NGC 1977 with the VLA at 330, 1420, 4860 and $8440 \mathrm{MHz}$. They detected the bright radio peak as an unresolved object designated as J0535-0452. The estimated flux density of the compact object is $320,50,11.4$ and $5.2 \mathrm{mJy}$ at $330,1420,4860$ and $8440 \mathrm{MHz}$ respectively. The spectrum of the object is straight, with no turn over down to $330 \mathrm{MHz}$. The spectral index is -1.3 over this range of frequencies. The $8440 \mathrm{MHz}$ observations are used to obtain an upper-limit on the source size of $\sim 100$ mas. The implied brightness temperature is $7 \times 10^{8} \mathrm{~K}$. Subrahmanyan et al. (2001) have concluded that the object is a non-thermal source.

Kutner et al. (1985) argued that the non-thermal source could be an extragalactic background source located behind NGC 1977. Compact steep spectrum extragalactic sources with

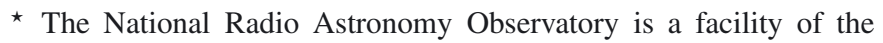
National Science Foundation operated under cooperative agreement by Associated Universities, Inc. spectral index close to -1.3 are typically high redshift objects ( 22 ; Miley \& De Breuck 2008). For these redshifts, the size of J0535-0452 is $\$ 780 \mathrm{pc}$, assuming standard cosmological parameters. Compact steep spectrum sources of this size typically show spectral turn over near a few $\mathrm{GHz}$, which is not the case for J0535-0452. Thus if J0535-0452 is an extragalactic source, then it should be one of the rare steep-spectrum, high redshift radio galaxies (for example, see Kaplan et al. 2000).

Another possibility is that the compact source in NGC 1977 is a pulsar. The spectral indices of both normal and millisecond pulsar are in the range -1 to -2.5 with a mean value of -1.65 (Karmer et al. 1998). Thus the compactness and spectral index of J0535-0452 may suggest that it is a pulsar. An earlier attempt to detect pulsed emission near $330 \mathrm{MHz}$ was not successful (Ramachandran 1995, unpublished). However, J0535-0452 would have eluded detection in earlier pulsar searches due to extreme scattering. We show in Sect. 2 that the best frequencies to search for pulsed emission from this pulsar are $\geq 5 \mathrm{GHz}$ depending on the pulsar period. We used the GBT to search for pulsed emission at frequencies near 4.8 and $14.8 \mathrm{GHz}$, but did not detect pulsed emission. The observations and results are given in Sects. 3 and 4, respectively.

\section{On observing pulsed emission from J0535-0452}

An attempt was made in 2003 to image J0535-0452 using the VLBA at $8.4 \mathrm{GHz}$ (Roshi et al. 2003, unpublished). The source was not detected in this observation. A possible interpretation of the non-detection in the VLBA observations is that the source would have angular broadened due to scattering in 


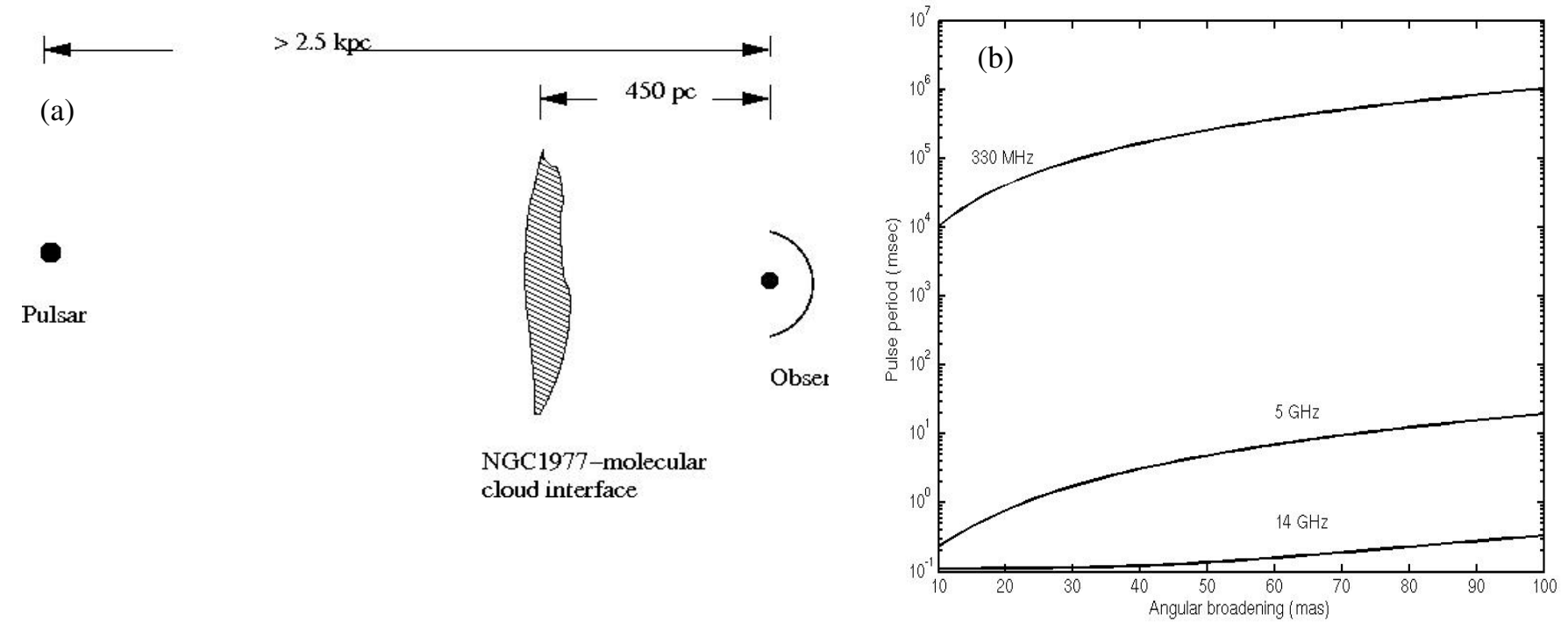

Fig. 1. a) Schematic showing the scattering geometry. The $\mathrm{H}_{\text {II }}$ region-molecular cloud interface (scattering screen) is located at $\sim 450 \mathrm{pc}$ from the observer and the distance to the compact source is $\geq 2.5 \mathrm{kpc}$. b) The expected pulse broadening for three frequencies $(330 \mathrm{MHz}, 5 \mathrm{and} 14 \mathrm{GHz})$ is shown in the pulsar period-angular broadening parameter space. The curves are the locus of points for pulse broadening equal to the pulsar period. For each frequency the parameter space above the curve can be probed by the pulsar search observation. The pulse broadening estimation includes contribution from an assumed intrinsic duty cycle of $10 \%$ of the pulsar period and broadening due to DM. For these calculation, the smallest pulsar period is taken as $1 \mathrm{~ms}$. The range of angular broadening is constrained by observations between 10 and $100 \mathrm{mas}$. For all frequencies $\lesssim 14 \mathrm{GHz}$, the pulse broadening is dominated by scattering. At $14 \mathrm{GHz}$, for angular broadening $\$ 60$ mas, the pulse broadening is limited by the assumed intrinsic duty cycle.

the $\mathrm{H}$ II region - molecular cloud interface. Assuming that the source is scatter broadened, a lower limit can be obtained for angular broadening which turns out to be $\sim 10$ mas. If J0535-0452 is a pulsar, then the pulsed emission will be broadened due to scattering in the $\mathrm{H}$ II region - molecular cloud interface. We estimate the pulse broadening using the measured limits on angular broadening of J0535-0452 along with the source-scattering screen geometry shown in Fig. 1a. The distance to the compact object, $D_{\text {pul }}$, as measured from $\mathrm{H}_{\mathrm{I}}$ absorption studies is $\geq 2.5 \mathrm{kpc}$ (Subrahmanyan et al. 2001) and the distance to NGC 1977, $D_{\text {hii }}$, is $\sim 450 \mathrm{pc}$ (Genzel et al. 1981). The $\mathrm{e}^{-1}$ pulse broadening time in sec due to scattering is obtained using the equation (Cordes \& Lazio 1997)

$t_{\mathrm{s}}=\frac{D_{\mathrm{hii}}}{D_{\text {pul }}-D_{\text {hii }}} \times \frac{D_{\text {pul }} \theta_{\mathrm{s}, 8.4 \mathrm{GHz}}^{2}}{8 \ln (2) c} \times\left(\frac{f_{\mathrm{GHz}}}{8.44}\right)^{-4}$,

where $c$ is the velocity of light in $\mathrm{cm} \mathrm{s}^{-1}$, the units of distances are in $\mathrm{cm}, f_{\mathrm{GHz}}$ is the observing frequency in $\mathrm{GHz}$ and $\theta_{\mathrm{s}, 8.4 \mathrm{GHz}}$ is the angular broadening at $8.4 \mathrm{GHz}$ in rad. The pulse broadening time is considered to scale with the 4 th power of frequency, appropriate for strong scattering. $\theta_{\mathrm{s}, 8.4 \mathrm{GHz}}$ range between 10 and 100 mas, which are obtained from the VLA (Subrahmanyan et al. 2001) and VLBA observations near $8.4 \mathrm{GHz}$ (Roshi et al. 2003, unpublished). In Fig. 1b we plot the estimated pulse broadening time for three observing frequencies $(330 \mathrm{MHz}, 5$ and $14 \mathrm{GHz}$ ) in the pulse period - angular broadening parameter space. The pulse broadening time in this plot also includes an assumed intrinsic duty cycle of $10 \%$ of the pulsar period (minimum pulsar period is taken as $1 \mathrm{~ms}$ ). The spectral resolution used for the calculation is $0.78 \mathrm{MHz}$. For a given dispersion measure (DM), this finite spectral resolution produces temporal broadening, which is also added to the plotted pulse broadening. Any temporal broadening due to DM griding in the pulsar search algorithm is neglected.
Continuum observations toward J0535-0452 can be used to estimate limits on the DM. The measured spectrum of the compact source does not show any turnover at $330 \mathrm{MHz}$ due to the continuum optical depth of the H II-region-molecular cloud interface. This fact is used to obtain an upper limit on the emission measure (EM) of $\sim 10^{4} \mathrm{~cm}^{-6}$ pc for an assumed ionized gas temperature of $8000 \mathrm{~K}$ and $\tau_{\mathrm{c}} \sim 0.05$ at $330 \mathrm{MHz}$. The 3 sigma uncertainty of the $330 \mathrm{MHz}$ map of NGC 1977 region (Subrahmanyan et al. 2001) is used to infer an upper limit on $\tau_{\mathrm{c}}$ of $\sim 0.05$. A DM corresponding to a given EM can be obtained as $D M=\sqrt{E M \phi L}$, where $\phi$ is the filling factor of the ionized gas in the intervening $\mathrm{H}$ II region and $L$ is the line of sight extent of the $\mathrm{H}$ II region. Taking $\phi \sim 1$ and $L \sim 10 \mathrm{pc}$, a good fraction of the size of the Orion molecular cloud, the DM we get is $\sim 300 \mathrm{pc} \mathrm{cm}^{-3}$. Contribution to DM due to the distributed ionized gas has to be added to the above estimate. NE2001 model (Cordes \& Lazio 2002) provides a total DM of $\sim 50 \mathrm{pc} \mathrm{cm}^{-3}$ in the direction of NGC 1977. Thus the upper limit obtained on $\mathrm{DM}$ is $\sim 350 \mathrm{pc} \mathrm{cm}^{-3}$. For estimating pulse broadening we used DM values up to $650 \mathrm{pc} \mathrm{cm}^{-3}$.

The result of the pulse broadening calculation shows that for all frequencies $\$ 14 \mathrm{GHz}$ the pulse broadening is dominated by scattering (see Fig. 1b). At $14 \mathrm{GHz}$, for $\theta_{\mathrm{s}, 8.4 \mathrm{GHz}}<60$ mas, pulse broadening plotted in Fig. $1 \mathrm{~b}$ is limited by the assumed intrinsic duty cycle. For each observing frequency indicated in Fig. 1b pulsed emission can be detected in the parameter space above the corresponding curve.

The estimated continuum flux density at $14.0 \mathrm{GHz}$ is $2.5 \mathrm{mJy}$. If the observed continuum emission is the mean flux density of the pulsar, then the pulsar can be detected at frequencies 5 and $14 \mathrm{GHz}$ (depending on the pulsar period) with signalto-noise ratio $>100$ in $1 \mathrm{~h}$ of observing time with the GBT. Thus, as seen in Fig. 1b, such an observation will be sensitive to both MSP and ordinary pulsars. Note that earlier searches would not have detected pulsars for the measured angular broadening limits (see Fig. 1b). 


\section{The GBT observations and data analysis}

The GBT observations on J0535-0452 were made at 4.8 and $14.8 \mathrm{GHz}$ on 15 May 2011. The GUPPI backend with $800 \mathrm{MHz}$ bandwidth, $1.6 \mathrm{MHz}$ spectral resolution and $41 \mu \mathrm{s}$ time resolution was used for the observations. Flux density calibration was done using the source 3C 161 for both frequencies. The flux densities of $3 \mathrm{C} 161$ were taken to be 6.7 and $2 \mathrm{Jy}$ at 4.8 and $14.8 \mathrm{GHz}$ (Ott et al. 1994). The measured telescope gains were 2 and $1.9 \mathrm{~K} / \mathrm{Jy}$ and system temperatures were 18.5 and $26 \mathrm{~K}$ at 4.8 and $14.8 \mathrm{GHz}$ respectively. Reference pointing was done on B0540-0415 at $14.8 \mathrm{GHz}$ and on 3C 161 at $4.8 \mathrm{GHz}$. The total on-source observing time at $14.8 \mathrm{GHz}$ is $27 \mathrm{~min}$ and that at $4.8 \mathrm{GHz}$ is $29 \mathrm{~min}$. The data processing was performed using PRESTO $^{1}$ (Ransom et al. 2002). A DM range of 0 to $720 \mathrm{pc} \mathrm{cm}^{-3}$ was searched with full time resolution. The value of $720 \mathrm{pc} \mathrm{cm}^{-3}$ is about two times larger than the upper limit on DM estimated in Sect. 2. In addition we searched for pulsar acceleration in the range 0 to $2.1 \times 10^{4} \times P \mathrm{~m} \mathrm{~s}^{-2}$, where $P$ is the period of the pulsar in sec.

\section{Results and conclusion}

We did not detect pulsed emission from J0535-0452. The upper limit obtained for pulsed emission at $4.8 \mathrm{GHz}$ is $55 \mu \mathrm{Jy}$ for a pulsar period of $10 \mathrm{~ms}$ and pulse broadening of $7 \mathrm{~ms}$ corresponding to an angular broadening of 60 mas (see Fig. 1b). At $14.8 \mathrm{GHz}$, the upper limit obtained is $30 \mu \mathrm{Jy}$ for a pulsar period of $3 \mathrm{~ms}$, typical for MSP, assuming 10\% intrinsic duty cycle.

Acceleration searches are most sensitive to binary pulsars with orbital period greater than 10 times the observation time
(Ransom et al. 2003). This means that our data analysis will rule out compact sources in binary system with orbital period $\gtrsim 5 \mathrm{~h}$ (assuming circular orbit). We have searched for accelerations up to $2.1 \times 10^{4} \times P \mathrm{~m} \mathrm{~s}^{-2}$. For orbital period of $\sim 5 \mathrm{~h}$, this upper limit rules out companion object of mass $\$ 3 M_{\odot}$ for an assumed pulsar period of $3 \mathrm{~ms}$. If the companion objects is of higher mass (i.e. $>3 M_{\odot}$ ) then for orbital periods $\gtrsim 5 \mathrm{~h}$ the object cannot be an ordinary star. This is because the orbital radius becomes comparable to the size of the star. The companion object could be a stellar mass black hole. However, we are not aware of any other observational evidence for the presence of a stellar mass black hole in the direction of NGC 1977 . Thus the only possibility that remains is that J0535-0452 is a binary MSP with short orbital period (i.e. $\$ 5 \mathrm{~h}$ ).

Acknowledgements. We thank the anonymous referee for the critical comments on the manuscript.

\section{References}

Cordes, J. M., \& Lazio, T. J. W. 1997, ApJ, 475, 557

Cordes, J. M., \& Lazio, T. J. W. 2002 [arXiv: astro-ph/0207156]

Genzel, R., Reid, M. J., Moran, J. M., \& Downes, D. 1981, ApJ, 244, 884

Ott, M., Witzel, A., Quirrenbach, A., et al. 1994, A\&A, 284, 331

Kaplan, D. L., Cordes, J. M., Condon, J. J., \& Djorgovski, S. G. 2000, ApJ, 529, 859

Karmer, M., Xilouris, K. M., Lorimer, D. R., et al. 1998, ApJ, 501, 270

Kutner, M. L., Machnik, D. E., Mead, K. N., \& Evans, N. J., II 1985, ApJ, 299, 351

Miley, G., \& De Breuck, C. 2008, A\&ARv, 15, 67

Ransom, S. M., Eikenberry, S. S., \& Middleditch, J. 2002, AJ, 124, 1788

Ransom, S. M., Cordes, J. M., \& Eikenberry, S. S. 2003, ApJ, 589, 911

Shaver, P. A., \& Goss, W. M. 1970, Aus. J. Phys. Astrophys. Suppl., 14, 133

Subrahmanyan, R., Goss, W. M., \& Malin, D. F. 2001, AJ, 121, 399

$\overline{{ }^{1} \text { http://www.nrao.edu/ sransom/presto }}$ 